\title{
Taste symmetry and QCD thermodynamics with improved staggered fermions
}

\author{
Alexei Bazavov ${ }^{* \dagger}$ and Peter Petreczky ${ }^{b}$ [for the HotQCD collaboration] $^{\S}$ \\ ${ }^{a}$ Department of Physics, University of Arizona, Tucson, AZ 85721, USA \\ ${ }^{b}$ Physics Department, Brookhaven National Laboratory, Upton, NY 11973, USA
}

\begin{abstract}
Taste symmetry violations in staggered fermion formulations correlate strongly with the cut-off (lattice spacing) dependence in thermodynamic quantities. Better taste symmetry on the lattice can be achieved either by decreasing the lattice spacing and going to larger temporal extent in finite-temperature calculations, or by further improving the action. The highly improved staggered quark (HISQ) action offers a further degree of improvement and substantially reduces taste violations. We report on our studies of the $2+1$ flavor QCD thermodynamics with the HISQ/tree action. By systematically comparing HISQ/tree, asqtad, p4 and stout calculations we quantify how the cut-off effects manifest themselves in different thermodynamic quantities, including the renormalized Polyakov loop, chiral condensate, various fluctuations and correlations of conserved charges. The implications for the equation of state and a comparison to the hadron resonance gas model are also discussed. The chiral aspects of the finite-temperature transition are discussed in the companion HotQCD contribution [1].
\end{abstract}

The XXVIII International Symposium on Lattice Filed Theory

June 14-19,2010

Villasimius, Sardinia, Italy

\footnotetext{
* Speaker.

${ }^{\dagger}$ Present address: Physics Department, Brookhaven National Laboratory, Upton, NY 11973, USA

¥This work has been supported in part by contracts DE-AC02-98CH10886 and DE-FC02-06ER-41439 with the U.S. Department of Energy and contract 0555397 with the National Science Foundation. The numerical calculations have been performed using the USQCD resources at Fermilab and JLab, the BlueGene/L at the New York Center for Computational Sciences (NYCCS) and the BlueGene/L at the Jülich Supercomputing Center. We thank Z. Fodor and S. Katz for sending us the stout data.

${ }^{\S}$ HotQCD Collaboration members are: A. Bazavov, T. Bhattacharya, M. Cheng, N.H. Christ, C. DeTar, S. Gottlieb, R. Gupta, P. Hegde, U.M. Heller, C. Jung, F. Karsch, E. Laermann, L. Levkova, C. Miao, R.D. Mawhinney, S. Mukherjee, P. Petreczky, D. Renfrew, C. Schmidt, R.A. Soltz, W. Soeldner, R. Sugar, D. Toussaint, W. Unger, P. Vranas
} 


\section{Introduction}

Improved staggered fermion formulations are widely used to study QCD at non-zero temperature and density [2]. Staggered fermions preserve a part of the chiral symmetry of continuum QCD and are inexpensive to simulate numerically. After staggering the fermion field the number of doublers is reduced from sixteen (in four dimensions) to four. These four species of fermions, that we call "tastes", are inequivalent at non-zero lattice spacing, because the taste symmetry is broken. It is restored in the continuum limit. To reduce the number of fermions further the rooting procedure is used. Its validity is discussed in Ref. [3].

Violations of the taste symmetry at non-zero lattice spacing contribute to the cut-off dependence of different physical observables. To reduce the taste violations, smeared links, i.e. weighted averages of different paths on the lattice that connect neighboring points, are used in the staggered Dirac operator. Various improved staggered fermion formulations, like p4, asqtad, stout and HISQ differ in the choice of smeared gauge links. The highly improved staggered quark (HISQ) action was developed by the HPQCD/UKQCD collaboration [ [ degree of improvement in the taste symmetry [ [ 4 , 5]. This action together with the one-loop tadpole improved gauge action is used by the MILC collaboration [5] to generate a library of ensembles with several lattice spacings and light quark masses. Such a combination of the fermion and gauge actions was originally referred to as HISQ. In our thermodynamic studies we use a tree-level improved gauge action. Thus, to distinguish our variant of the action from the one that MILC and HPQCD originally used we call it the HISQ/tree action.

In this contribution we extend our studies [6] of $2+1$ flavor QCD thermodynamics with the HISQ/tree action, compare with the results obtained with p4, asqtad and stout and discuss the discretization effects in different thermodynamic quantities.

\section{Taste symmetry of different actions}

We have generated a library of zero- and finite-temperature HISQ/tree ensembles along two lines of constant physics (LCP): $m_{l}=m_{s} / 5$ and $m_{l}=m_{s} / 20$, where $m_{l}\left(m_{s}\right)$ is the light (strange) quark mass. The parameters of these ensembles have been reported in [6]. For $m_{s} / 5$ our lattice spacings range from $a=0.15 \mathrm{fm}$ to $0.23 \mathrm{fm}$, and for $m_{s} / 20$ from $0.08 \mathrm{fm}$ to $0.23 \mathrm{fm}$. The statistics has been updated and the current status is shown in Table 1.

Violations of the taste symmetry lead to splitting of the staggered pion spectrum into sixteen pion states grouped into eight multiplets, that we label in the taste basis as $\gamma_{5}, \gamma_{0} \gamma_{5}, \gamma_{i} \gamma_{5}, \gamma_{i} \gamma_{j}, \gamma_{i} \gamma_{0}$, $\gamma_{i}, \gamma_{0}$ and 1. $\gamma_{5}$ corresponds to the Goldstone pion, the lowest state whose mass approaches zero in the chiral limit, while the masses of the other tastes always stay finite at non-zero lattice spacing. In the continuum the pion spectrum becomes degenerate, and the difference in mass between tastes vanishes. We denote the quadratic splittings as $\Delta_{\alpha}=m_{\alpha}^{2}-m_{\gamma_{5}}^{2}$, where $\alpha$ is one of the above tastes except $\gamma_{5}$.

In earlier work [6] we measured the pion splittings on the ensembles with the light quark mass of $m_{s} / 5$. Zero-temperature ensembles along this LCP were limited to lattices down to $a=0.15$ $\mathrm{fm}$. To study the behaviour of the spectrum at smaller lattice spacings we measured $\Delta_{\alpha}$ using the valence light quark mass of $m_{s} / 5$ on zero-temperature ensembles with the light sea quark mass of 


\begin{tabular}{|l|l|l||l|r||r|r|}
\hline \multicolumn{2}{|c||}{} & \multicolumn{2}{c||}{$T=0$} & \multicolumn{2}{c|}{$T>0$} \\
\hline$\beta$ & $r_{0} / a$ & $a m_{s}$ & $N_{s}^{3} \times N_{\tau}$ & $\mathrm{TU}$ & $\mathrm{TU}, N_{\tau}=8$ & $\mathrm{TU}, N_{\tau}=6$ \\
\hline 6.000 & $2.094(21)$ & 0.1138 & $24^{3} \times 32$ & 2,305 & & 6,020 \\
6.050 & $2.198^{*}$ & 0.1064 & $24^{3} \times 32$ & 2,675 & & 6,340 \\
6.075 & $2.253^{*}$ & 0.1036 & & & & 4,700 \\
6.100 & $2.310^{*}$ & 0.0998 & $28^{3} \times 32$ & 1,875 & & 6,280 \\
6.125 & $2.368^{*}$ & 0.0966 & & & & 9,990 \\
6.150 & $2.427^{*}$ & 0.0936 & & & & 11,220 \\
6.175 & $2.489^{*}$ & 0.0906 & & & & 10,860 \\
6.195 & $2.531(24)$ & 0.0880 & $32^{4}$ & 2,365 & 9,990 & 11,100 \\
6.215 & $2.589^{*}$ & 0.0862 & & & & 6,390 \\
6.245 & $2.668^{*}$ & 0.0830 & & & 1,600 & 6,410 \\
6.260 & $2.707^{*}$ & 0.0810 & & & 1,790 & \\
6.285 & $2.775^{*}$ & 0.0790 & $32^{4}$ & 2,300 & 6,190 & 6,750 \\
6.315 & $2.858^{*}$ & 0.0760 & & & 1,805 & \\
6.341 & $2.932^{*}$ & 0.0740 & $32^{4}$ & 1,325 & 7,020 & 6,590 \\
6.354 & $2.986(41)$ & 0.0728 & $32^{4}$ & 2,295 & 5,990 & 5,990 \\
6.390 & $3.076^{*}$ & 0.0694 & & & 8,760 & \\
6.423 & $3.189(22)$ & 0.0670 & $32^{4}$ & 2,295 & 5,990 & 5,990 \\
6.460 & $3.292^{*}$ & 0.0640 & $32^{3} \times 64$ & 1,030 & 10,990 & \\
6.488 & $3.395(31)$ & 0.0620 & $32^{4}$ & 2,295 & 11,990 & 8,790 \\
6.515 & $3.470^{*}$ & 0.0604 & $32^{4}$ & 2,045 & 10,100 & 10,430 \\
6.550 & $3.585(14)$ & 0.0582 & $32^{4}$ & 2,295 & 11,990 & 7,270 \\
6.575 & $3.674^{*}$ & 0.0564 & $32^{4}$ & 2,295 & 14,500 & 7,330 \\
6.608 & $3.765(23)$ & 0.0542 & $32^{4}$ & 2,295 & 11,990 & 6,560 \\
6.664 & $3.994(14)$ & 0.0514 & $32^{4}$ & 2,295 & 11,990 & 8,230 \\
6.800 & $4.568(30)$ & 0.0448 & $32^{4}$ & 2,295 & 11,990 & 7,000 \\
6.950 & $5.187(39)$ & 0.0386 & $32^{4}$ & 2,295 & 11,990 & 7,480 \\
7.150 & $6.186(72)$ & 0.0320 & $32^{4}$ & 2,295 & 11,990 & 4,770 \\
\hline
\end{tabular}

Table 1: The parameters of the numerical simulations: gauge coupling, strange quark mass and the number of time units (TU) for each run. The lattices are separated by $5 \mathrm{TU}$ at zero and $10 \mathrm{TU}$ at finite temperature. The volumes for zero-temperature runs are given in column 4 . At finite temperature we used $32^{3} \times 8$ and $24^{3} \times 6$ volumes. In the second column the values with error bars represent $r_{0} / a$ determination from the static quark anti-quark potential and the values marked by asterisk $(*)$ are determined from an Allton-style fit. The lattice spacing is determined by using $r_{0}=0.469 \mathrm{fm}$.

$m_{s} / 20$. This is justified because quadratic splittings do not depend on the quark mass at the lowest order in the chiral perturbation theory, and also, as the measurements with valence HISQ on asqtad sea have shown, $\Delta_{\alpha}$ are sensitive to the "roughness" of configurations and not to the details of the action used to generate them. The results are presented in Fig. 1. The vertical line separates our previously published [6] data (to the right) and the recently added calculations (to the left). The stout data for $\Delta_{\gamma_{i} \gamma_{5}}$ and $\Delta_{\gamma_{i} \gamma_{j}}$ are shown as open symbols. We have also extracted approximate values of the stout $\Delta_{\gamma_{i}}$ and $\Delta_{1}$ from the right panel of Fig. 2 in Ref. [7], and assumed for stout $\Delta_{\gamma_{0} \gamma_{5}}=\Delta_{\gamma_{i} \gamma_{5}}, \Delta_{\gamma_{i} \gamma_{0}}=\Delta_{\gamma_{i} \gamma_{j}}$ and $\Delta_{\gamma_{0}}=\Delta_{\gamma_{i}}$. When plotted against $a^{2}$, we expect the splittings to show some curvature for all actions, because in the scaling regime we expect them to scale with $\alpha_{s}^{2} a^{2}$ for asqtad and HISQ/tree, and with $\alpha_{s} a^{2}$ for stout. We found that $\Delta_{\alpha}$ for different actions can be fit to a polynomial form $f\left(a^{2}\right)=B_{2} a^{2}+B_{4} a^{4}+B_{6} a^{6}$ up to the lattice spacing $a=0.22 \mathrm{fm}$. After 


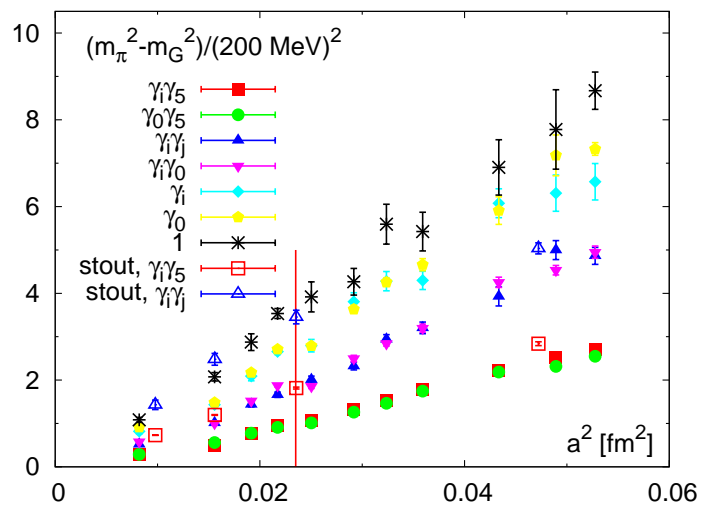

Figure 1: The splitting between different pion multiplets calculated for HISQ/tree, $m_{G} \equiv m_{\gamma_{5}}$.

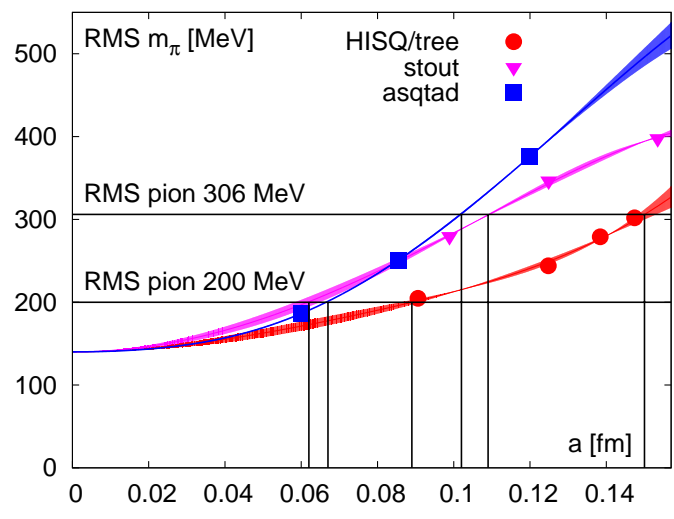

Figure 2: RMS pion mass when $m_{\gamma_{5}}=140 \mathrm{MeV}$. See details in the text.

performing such fits, we obtained the splittings $\Delta_{\alpha}$ as functions of $a^{2}$ for HISQ/tree, asqtad and stout.

Having data for the splittings we set the Goldstone pion mass to $m_{\gamma_{5}}=140 \mathrm{MeV}$ and calculate the root-mean-squared (RMS) pion mass as a function of the lattice spacing:

$$
m_{\pi}^{R M S}=\sqrt{\frac{1}{16}\left(m_{\gamma_{5}}^{2}+m_{\gamma_{0} \gamma_{5}}^{2}+3 m_{\gamma_{i} \gamma_{5}}^{2}+3 m_{\gamma_{i} \gamma_{j}}^{2}+3 m_{\gamma_{i} \gamma_{0}}^{2}+3 m_{\gamma_{i}}^{2}+m_{\gamma_{0}}^{2}+m_{1}^{2}\right)} .
$$

The results are presented in Fig. 2. Curves show the RMS pion mass obtained from fitted values of $\Delta_{\alpha}$. The thickness of each band represents the systematic error introduced by varying the end of the fitting interval from 0.17 to $0.22 \mathrm{fm}$. The symbols correspond to the RMS pion calculated from the measured pion splittings. For our estimates below we took the midpoints in each band.

Consider lattice spacing $a=0.15 \mathrm{fm}$. On an $N_{\tau}=8$ lattice it corresponds to temperature $T=164 \mathrm{MeV}$, well in the transition region. At this $a$ the RMS pion mass is $306 \mathrm{MeV}$ for $\mathrm{HISQ} /$ tree, $394 \mathrm{MeV}$ for stout, and $496 \mathrm{MeV}$ for asqtad. Having the same mass as for HISQ/tree requires $a=0.102 \mathrm{fm}$ for asqtad and $a=0.109$ for stout. In other words, a HISQ/tree simulation on $N_{\tau}=8$ at $T=164 \mathrm{MeV}$ is comparable to an asqtad simulation on $N_{\tau}=197.3 / 164 / 0.102 \simeq 11.8$, or a stout simulation on $N_{\tau}=197.3 / 164 / 0.109 \simeq 11.0$ at the same $T$. Thus, we expect HISQ/tree $N_{\tau}=8$ results to be close to stout $N_{\tau}=10$ and asqtad $N_{\tau}=12$ results, as far as the taste symmetry is concerned. For comparison, if one desires to have $200 \mathrm{MeV}$ RMS pion at $T=164 \mathrm{MeV}$, this requires $a=0.089 \mathrm{fm}$ for HISQ/tree, $a=0.067 \mathrm{fm}$ for asqtad, and $a=0.062 \mathrm{fm}$ for stout. This translates into the temporal extent of $N_{\tau} \simeq 13.5,18.0,19.4$, respectively, for these actions. The lattice spacings discussed above are represented with vertical lines in Fig. 2. These estimates are rather crude, but are completely in line with the conclusion of [5] that a HISQ (or HISQ/tree) ensemble with spacing $a$ is comparable to an asqtad ensemble with $2 / 3 a$.

Lattice artifacts, related to taste symmetry breaking, affect masses of hadron states, and, in general, distort the hadron spectrum. In our simulations the masses of the pseudoscalar mesons $m_{\pi}$ and $m_{K}$ were used as input to constrain the LCP. However, other states, e.g. vector mesons or baryons, are predictions and can show how well the spectrum can be reproduced at a given lattice spacing. In Fig. 3 we present masses of $\rho, K^{*}, \phi$ mesons, nucleon and $\Omega$-baryon along with 

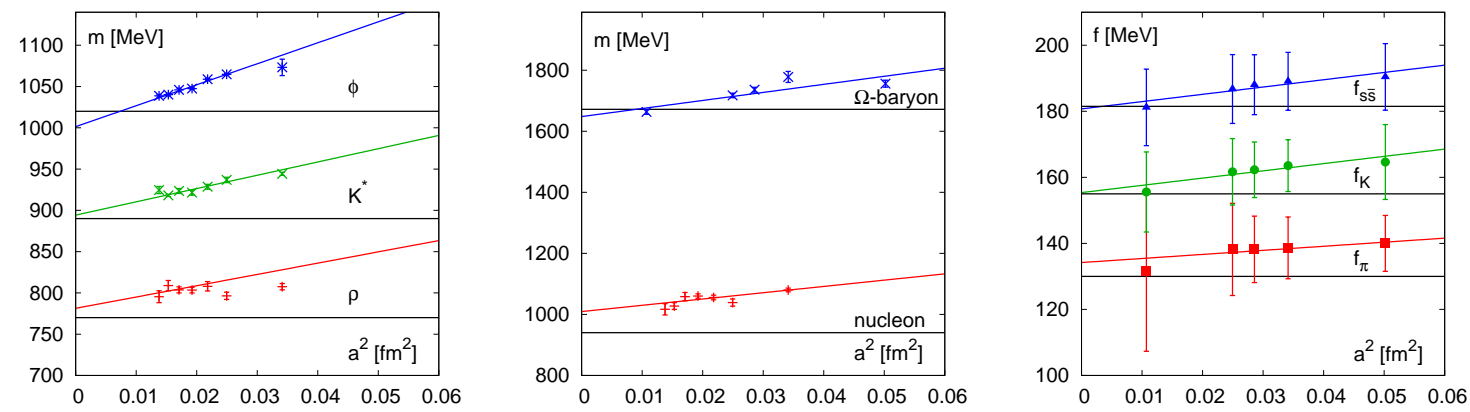

Figure 3: Preliminary results for masses of vector mesons (left), nucleon and $\Omega$ (middle) and the decay constants of pseudoscalar mesons, including the fictitious $\eta_{s \bar{s}}$ meson (right) for HISQ/tree, $m_{l}=m_{s} / 20$.
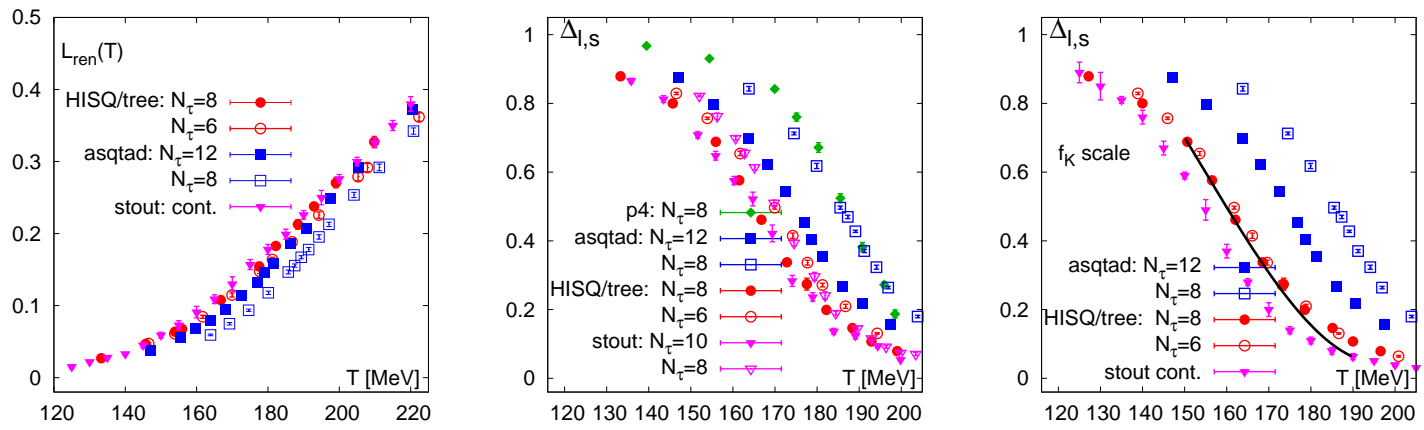

Figure 4: The Polyakov loop (left), chiral condensate with $r_{0}$ (middle) and with $f_{K}$ (right) scale.

$a^{2}$ extrapolations to the continuum. We have also calculated the pseudoscalar decay constants. Preliminary results for five ensembles are shown in the rightmost panel of Fig. 3.

\section{Taste symmetry and thermodynamic quantities}

Now we turn to the discussion of different thermodynamic quantities. In the left panel of Fig. 4 the renormalized Polyakov loop is shown for several actions. This quantity is associated with deconfinement and is not very sensitive to the chiral properties of the action. Therefore we observe mild effects from going to finer lattices or to actions having less taste breaking. The chiral condensate, shown in the middle panel of Fig. 4, on the contrary, exhibits significant differences for $\mathrm{p} 4$, asqtad and HISQ/tree on lattices with the same $N_{\tau}=8$. We attribute these to the amount of taste breaking that these actions have. P4 and asqtad, having higher degree of taste breaking, operate at a higher RMS pion mass than HISQ/tree and stout; therefore the transition is shifted to higher temperature. As shown in Sec. 2 at $T=164 \mathrm{MeV}$ the asqtad RMS pion mass is about $496 \mathrm{MeV}$, and the HISQ/tree mass is about $306 \mathrm{MeV}$. This improvement in the taste symmetry shifts the transition region by about $15 \mathrm{MeV}$. (Compare HISQ/tree and asqtad $N_{\tau}=8$ curves in Fig. \#.) The stout data in the middle panel of Fig. $\bigoplus$ is plotted by converting the $f_{K}$ scale to $r_{0}$ scale (using $f_{K}$ and $r_{0}$ values calculated by the Budapest-Wuppertal collaboration). This allows us to compare the data for different actions at finite lattice spacing, when the latter is set by the same procedure.

To directly compare to the results of the Budapest-Wuppertal group [7] we have also plotted the chiral condensate with the temperature scale set by $f_{K}$ (for HISQ/tree, but not asqtad) rather 


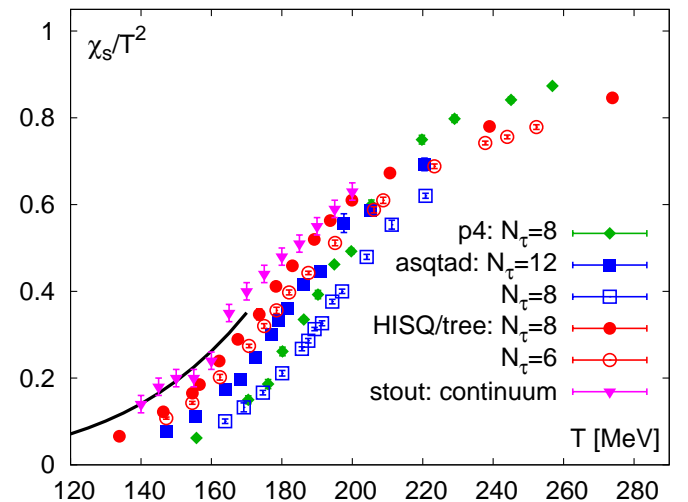

Figure 5: The strange quark number susceptibility. The solid line represents the HRG model.

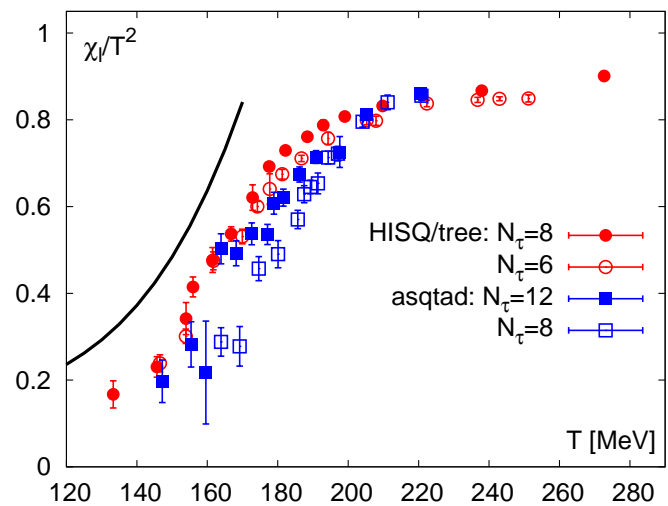

Figure 6: The light quark number susceptibility. The solid line represents the HRG model.

than $r_{0}$. This is shown in the right panel of Fig. 4 . As one can see, HISQ/tree $N_{\tau}=6$ and 8 results collapse into one curve which is almost identical with the solid curve that represents the $\mathrm{HISQ} /$ tree continuum result extrapolated from $N_{\tau}=6$ and 8 using the $r_{0}$ scale. We attribute the difference between the HISQ/tree and stout continuum extrapolations to the light quark mass difference: HISQ/tree (and asqtad) LCP uses $m_{l}=m_{s} / 20$, while stout LCP is tuned to $m_{l}=m_{s} / 27$.

We also study the strange and light quark number susceptibilities, Fig. 5 and 6 . These quantities are associated with deconfinement and signal the liberation of degrees of freedom with certain quantum numbers. Because these quantities are related to hadron properties in the low temperature region, they are sensitive to the amount of taste breaking at finite lattice spacing. In line with previous observations the asqtad data at finite lattice spacing is shifted to higher temperatures, compared to HISQ/tree. The strangeness-baryon number correlations are shown in Fig. 7. The agreement between the HRG model and the lattice is considerably improved with the HISQ/tree action. We conclude from Fig. 7 that this quantity is dominated by the states heavy enough, so that their masses are reproduced well with the HISQ/tree action even at finite lattice spacing, corresponding to $N_{\tau}=6$ and higher.

Finally, we discuss the effects of the taste symmetry breaking on the trace anomaly, Fig. 8 . In the low-temperature region $(T<160 \mathrm{MeV})$ our HISQ/tree results agree well with the continuum estimate for the stout data, obtained by Budapest-Wuppertal Collaboration by averaging over $N_{\tau}=6$ and 8 or $N_{\tau}=8$ and 10, respectively (see [8] for details), but not with the p4 and asqtad results. This is expected, again, due to higher degree of taste breaking in $\mathrm{p} 4$ and asqtad. At high temperatures $(T>250 \mathrm{MeV}) \mathrm{p}$, asqtad and HISQ/tree results agree between themselves, but significantly disagree with the stout data. We note that $\mathrm{p} 4$, asqtad, and HISQ/tree actions improve the quark dispersion relation whereas the stout action (with conventional staggered fermions) does not. The solid line in Fig. 8 is a parametrization of the equation of state derived in [9] based on the hadron resonance gas model and lattice data.

\section{Conclusion}

Taste symmetry breaking at finite lattice spacing is one of the major cut-off effects in staggered 


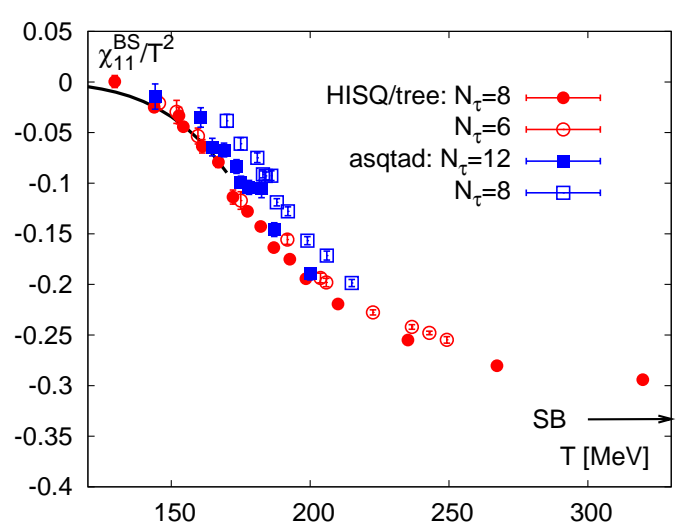

Figure 7: The $S-B$ correlations.

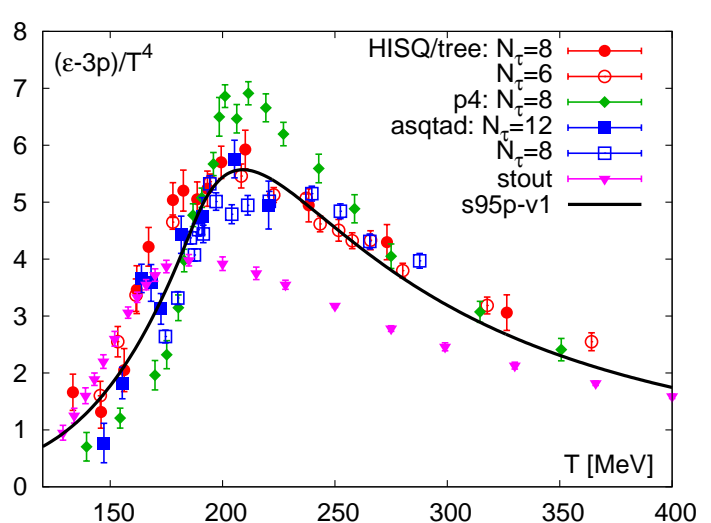

Figure 8: The trace anomaly. See details in the text.

actions. By comparing several staggered actions with a different degree of improvement and at different lattice spacings, we studied the cut-off dependence of several thermodynamic quantities. As expected, the quantites that are either directly related to the chiral symmetry (such as the chiral condensate) or are sensitive to the hadron spectrum (for instance, quark number susceptibilities and the interaction measure) are affected by taste symmetry breaking of the staggered fermion actions. The HISQ action, having the highest degree of improvement, exhibits the smallest cut-off effects, compared to $\mathrm{p} 4$, asqtad and stout.

\section{References}

[1] W. Söldner [HotQCD Collaboration], PoS (LAT2010) 215

[2] C. E. DeTar, PoS (LAT2008) 001 [arXiv:0811.2429 [hep-lat]]; P. Petreczky, Nucl. Phys. A 830, 11C (2009) [arXiv:0908.1917 [hep-ph]]; P. Petreczky, Nucl. Phys. Proc. Suppl. 140, 78 (2005) [arXiv:hep-lat/0409139].

[3] S. R. Sharpe, PoS (LAT2006) 022 [arXiv:hep-lat/0610094]; M. Creutz, BoS (LAT2007) 007 [arXiv:0708.1295 [hep-lat]].

[4] E. Follana et al. [HPQCD collaboration and UKQCD collaboration], Phys. Rev. D 75 (2007) 054502 [arXiv:hep-lat/0610092].

[5] A. Bazavov et al. [MILC collaboration], Phys. Rev. D 82, 074501 (2010) [arXiv:1004.0342 [hep-lat]].

[6] A. Bazavov and P. Petreczky [HotQCD Collaboration], PoS (LAT2009) 163 [arXiv:0912.5421 [hep-lat]]; A. Bazavov and P. Petreczky [HotQCD collaboration], J. Phys. Conf. Ser. 230, 012014 (2010) [arXiv:1005.1131 [hep-lat]].

[7] S. Borsanyi, Z. Fodor, C. Hoelbling, S. D. Katz, S. Krieg, C. Ratti and K. K. Szabo [Wuppertal-Budapest Collaboration], JHEP 1009, 073 (2010) [arXiv:1005.3508 [hep-lat]].

[8] S. Borsanyi et al., arXiv:1007.2580 [hep-lat].

[9] P. Huovinen and P. Petreczky, Nucl. Phys. A 837, 26 (2010) [arXiv:0912.2541 [hep-ph]]. 